\title{
Archives Of Breast

DOI: 10.32768/abc.202291119-122

\section{A Rare Case of Lobular Carcinoma in Situ Within a Fibroadenoma}

\author{
Fui Tin Pang ${ }^{\star a, b}$, Anat Kornecki ${ }^{\mathrm{b}}$, Kalan Lynn ${ }^{\mathrm{b}}$, Sze Yuen Lee \\ aHospital Queen Elizabeth II, Sabah, Malaysia \\ ${ }^{b}$ Department of Medical Imaging, Western University, St Joseph's Health Care London, Ontario, Canada \\ ${ }^{c}$ Department of Radiology, Hospital Segamat, Johor, Malaysia
}

ARTICLE INFO

Received:

06 June 2021

Revised:

18 October 2021

Accepted:

09 November 2021

Keywords:

Breast Carcinoma in Situ,

Fibroadenoma,

Breast Neoplasms

Copyright (C) 2022. This is an open-access article distributed under the terms of the Creative Commons Attribution-Non-Commercial 4.0 International License, which permits copy and redistribution of the material in any medium or format or adapt, remix, transform, and build upon the material for any purpose, except for commercial purposes.

\section{INTRODUCTION}

Fibroadenoma (FA), a biphasic tumor, e.g., a tumour composed of integral epithelial and stromal components, is the most common benign solid breast tumor in the adolescent population. It is generally managed as a BI-RADS 3 lesion with short-term (3-6 months) follow up as recommended in the ACR BI-RADS guideline. Though unusual, there is a very small number of FAs that harbor carcinoma or high-risk lesions with reported incidence of as low as $0.002 \%$ to $0.125 \%$. Among these, 50\% were lobular carcinoma in situ (LCIS) and 20\% were ductal carcinoma in situ (DCIS).$^{1-7}$ In this case report, we present a rare case of LCIS within a FA.

\footnotetext{
*Address for correspondence:

Fui Tin Pang, MD

B-3-1, Maya Condo, Jalan Bunga Matahari 1, 88400, Kota

Kinabalu, Sabah, Malaysia

Tel: +6016 8880154

Email: pangfuitin@gmail.com
}

\section{CASE PRESENTATION}

A 43-year-old woman, who had a history of bilateral stable benign-appearing breast masses, presented with new symptoms of right-sided intermittent, non-spontaneous brownish-colored nipple discharge. Bilateral 2 dimension (2D) and 3 dimension (3D) mammographic views were obtained, showing a developing right breast mass in the lower outer quadrant, anterior third (Figures 1 and 2). No associated calcifications or distortion were identified. There were no abnormalities in the left breast. Targeted ultrasound (US) was performed, demonstrating within the corresponding region an oval-shaped hypoechoic mass with lobulated margin along the 8 o'clock radian, at the retroareolar complex, measuring $2.0 \times 1.3 \times 1.8 \mathrm{~cm}$ (Figure 3a). An associated moderate internal vascularity was detected on color Doppler flow (Figure 3b). As this was a new mammographic finding and given the sonographic morphology, further assessment with core biopsy was performed. The biopsy pathology 
report showed a biphasic fibroepithelial lesion and a diagnosis of phyllodes could not be excluded. Given the imaging presentation and the inconclusive pathology results, the patient was referred for surgical consultation after which a surgical excision with the guidance of hook wire localization was performed (Figure 4). Surgical pathology results showed classic

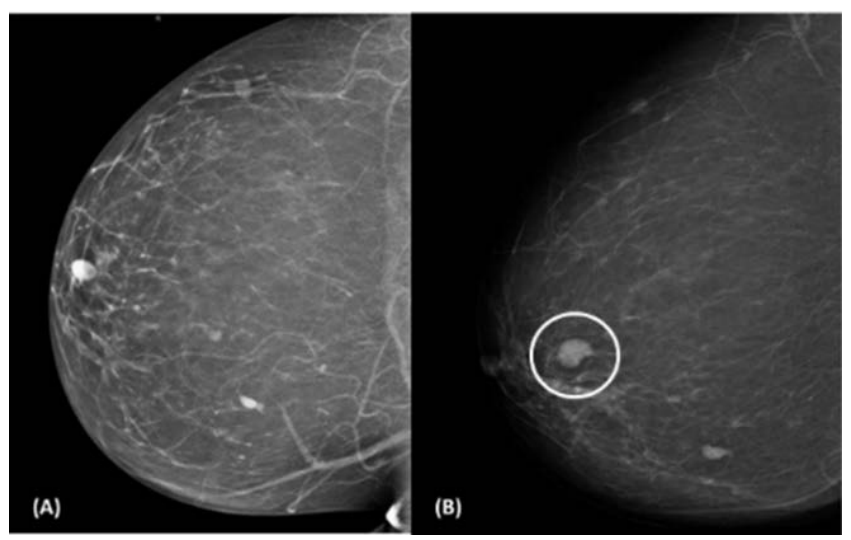

Figure 1. Right CC mammogram views done, A. in 2018 and B. in 2020. White circle shows the developing right lower outer breast mass which was later biopsied with pathology results of FA containing LCIS within the lesion and in adjacent breast tissue
LCIS within a FA with involvement of the adjacent breast tissue (e-cadherin negative). The patient was then placed on Tamoxifen as chemo-prevention for five years with annual screening mammography and was discharged to the care of her family doctor four months after her lumpectomy. Clinical follow-up was unremarkable with resolution of the nipple discharge.

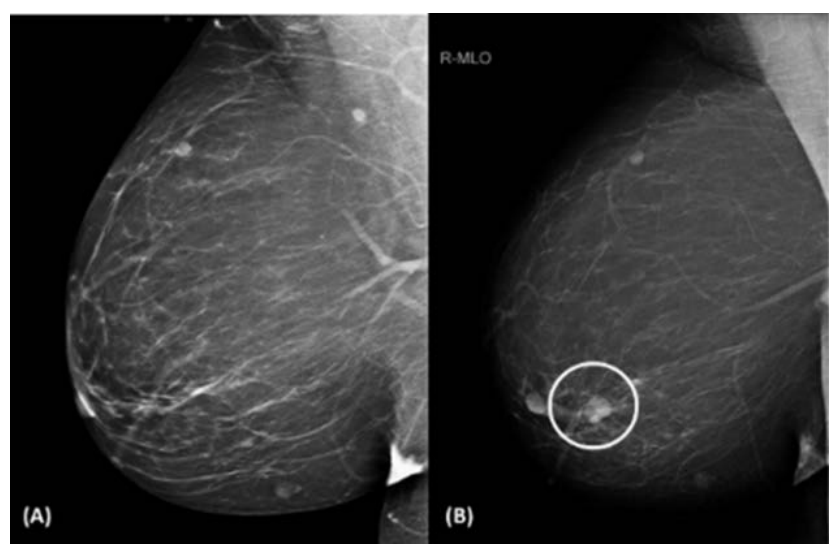

Figure 2. Right MLO mammogram views done, A. in 2018 and B. and 2020. White circle shows the developing right lower outer breast mass.

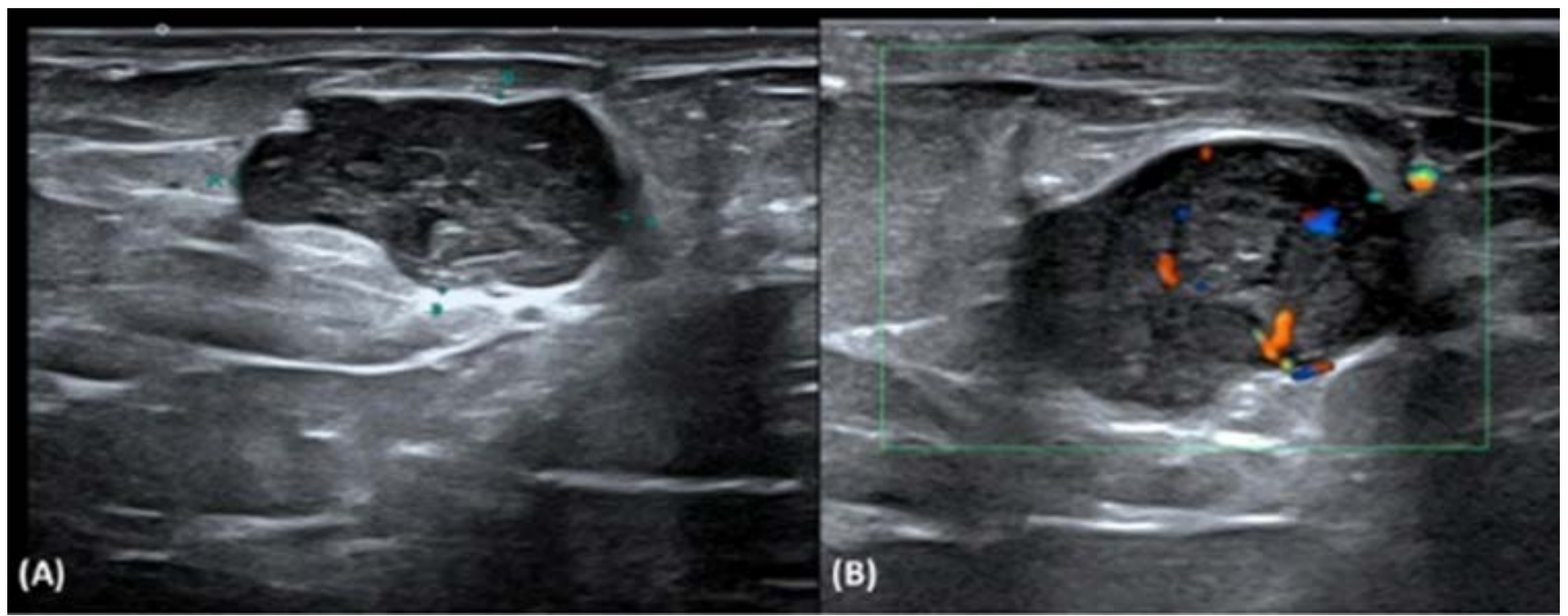

Figure 3. A. Right breast US scan confirmed a new solid hypoechoic mass along the 8 o'clock radian, corresponding to the mammographic finding. B: Color Doppler US demonstrated focal increased vascularity within the right breast 8 o'clock radian mass.

\section{DISCUSSION}

FA, a biphasic tumor, is a common benign solid breast tumor with peak incidence in the adolescent population. It is documented that FAs account for $68 \%$ of all breast masses and up to 94\% of biopsied breast lesions. ${ }^{1,8} \mathrm{FAs}$ that are larger than $5 \mathrm{~cm}$ are categorized as giant FAs. They can occur anywhere in the breast but are found predominantly in the upper outer quadrant. $^{2}$ Histologically and radiologically, FAs can be subdivided into simple and complex. Complex FAs are associated with an increased risk of breast cancer and may include cysts $(>3 \mathrm{~mm})$, sclerosing adenosis, epithelial calcification or papillary apocrine change. ${ }^{9,10}$ Carcinomas and high-risk lesions within FAs can either arise from FAs and be entirely encased by the FA or can focally involve both the FA and the adjacent tissue. In our case, the patient falls under the latter. 


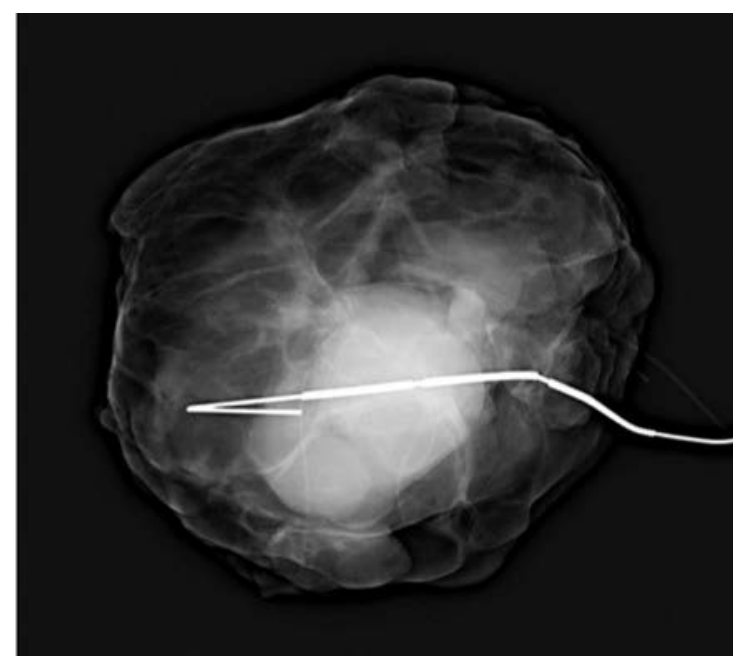

Figure 4. Lumpectomy specimen x-ray demonstrated the presence of both the mass and penetrating hookwire.

In the final pathology report, the microscopic description stated that, that sections showed a fibroepithelial lesion with focal leaf-like architecture in which cellularity was low and there was no evidence of stromal overgrowth. There was no significant cytologic atypia and no mitotic figures identified, features consistent with those of a FA. Thus, phyllodes tumor was excluded. Focally, the FA and adjacent breast tissue was involved by LCIS (ecadherin negative).

Several studies have reported that FAs with associated carcinomas and high-risk lesions occur mainly in patients 10-20 years older than the typical mean age of simple FAs. ${ }^{4,11}$ There is no classic clinical presentation and nipple-discharge is not common for either FAs or LCIS. Our patient at her forties complained of brownish nipple-discharge which could be related to the location of the lesion within the retroareolar margin. Nevertheless, this was resolved after the surgery.

LCIS is lobulocentric proliferation of monomorphic cells which fill up and distend most of the acini in the involved lobule whereas atypical lobular hyperplasia (ALH) does not distend the acini but both are considered as lobular neoplasm. Until today, no reliable radiologic features of lobular neoplasm within FAs have been reported. There are several studies showing that LCIS may be associated with a subtle area of increased color flow signals demonstrated on color Doppler study. ${ }^{3,11}$ Retrospectively, our patient did demonstrate focal increased color flow signal within the FA but this was not considered to be a specific finding as any benign cellular tumors may give rise to similar features. Presence of these lobular neoplasms within FAs is normally identified incidentally after a lumpectomy for a suspected benign lesion as there are no pathognomonic imaging findings. In this case, our patient presented in her forties with a new developing mammographic mass with increased internal flow as well as lobulated margin on US examination. It was these features that prompted the radiologist to be more alert about the fact that this may not be a simple FA.

Nowadays, LCIS is no longer considered precancerous to invasive carcinoma but a risk factor for breast cancer. ${ }^{12,13}$ It has also been reported that both LCIS found independently and LCIS within FAs to have similar biological behavior and prognosis. ${ }^{11}$ Hence, recent reports have recommended managing these lesions as non-malignant lesions predicting increased bilateral breast cancer risk, using close monitoring as well as hormonal chemoprevention. However, these do not apply to the variants of LCIS especially pleomorphic LCIS and LCIS with necrosis as these are considered aggressive types of LCIS. Studies have shown that these variants have upgrade rates as high as $27 \%$ after core biopsy which is why they are warranted for surgical excision. ${ }^{12,14}$ Supplementary screening in addition to routine mammography might be required for future follow-up. Our patient was put on Tamoxifen as chemoprevention for five years with annual screening mammography. To date, there are no official guidelines for the management of LCIS in the authors' countries, Malaysia and Canada.

\section{CONCLUSION}

Despite being considered benign, FAs should still be closely monitored by US as they can still harbor in situ or invasive carcinomas or high-risk lesions, including LCIS. The radiologists should always be aware and consider these as part of their differential diagnosis whenever benign-looking masses have atypical features or presentation. The current management of LCIS detected within FA is still similar to LCIS found elsewhere in the breast. Though LCIS is no longer considered pre-cancerous, its diagnosis is important as it is associated with increased risk of bilateral breast cancer.

\section{ETHICAL CONSIDERATIONS}

The patient gave written informed consent to publish her information and images contained within this case report.

\section{CONFLICT OF INTEREST}

The authors declare that they have no conflict of interest related to the publication of this manuscript. 


\section{REFERENCES}

1. Cerrato F, Labow BI. Diagnosis and management of fibroadenomas in the adolescent breast. Semin Plast Surg. 2013;27(1):23-5. doi: 10.1055/s-0033-1343992.

2. Foster ME, Garrahan N, Williams S. Fibroadenoma of the breast: a clinical and pathological study. J R Coll Surg Edinb. 1988;33(1):16-9. doi: Not available.

3. Brock CM, Harper C, Tyler T. Fibroadenoma containing lobular carcinoma in situ, an unusual finding in a normally benign mass. J Surg Case Rep. 2020;2020(4):rjaa059. doi: 10.1093/jscr/rjaa059.

4. Diaz NM, Palmer JO, McDivitt RW. Carcinoma Arising Within Fibroadenomas of the Breast A Clinicopathologic Study of 105 Patients. American Journal of Clinical Pathology. 1991;95(5):614-22. doi: 10.1093/ajcp/95.5.614.

5. Greenberg R, Skornick Y, Kaplan O. Management of breast fibroadenomas. Journal of General Internal Medicine. 1998;13(9):640-5. doi: 10.1046/j.15251497.1998.cr188.x.

6. Wu Y-T, Chen S-T, Chen C-J, Kuo Y-L, Tseng L-M, Chen D-R, et al. Breast cancer arising within fibroadenoma: collective analysis of case reports in the literature and hints on treatment policy. World Journal of Surgical Oncology. 2014;12(1). doi: 10.1186/14777819-12-335.

7. El-Essawy M, Al Haidary A, Khan AL. Ductal carcinoma in situ (DCIS) in breast fibroadenoma. Egyptian Journal of Radiology and Nuclear Medicine. 2020;51(1). doi: 10.1186/s43055-020-00191-5.

8. Lee $M$, Soltanian HT. Breast fibroadenomas in adolescents: current perspectives. Adolesc Health Med Ther. 2015;6:159-63. doi: 10.2147/AHMT.S55833.
9. Hua B, Xu JY, Jiang L, Wang Z. Fibroadenoma with an unexpected lobular carcinoma in situ: A case report and review of the literature. Oncol Lett. 2015;10(3):1397-401. doi: 10.3892/ol.2015.3488.

10. Santen RJ. Benign Breast Disease in Women. [Updated 2018 May 25]. In: Feingold KR, Anawalt B, Boyce A, et al., editors. Endotext [Internet]. South Dartmouth (MA): MDText.com, Inc.; 2000-. Available at: https://www.ncbi.nlm.nih.gov/books/NBK278994/

11. Tiu CM, Chou YH, Chiou SY, Hsu CY, Chen SP, Chiang HR, et al. Development of a carcinoma in situ in a fibroadenoma: color Doppler sonographic demonstration. J Ultrasound Med. 2006;25(10):13358. doi: 10.7863/jum.2006.25.10.1335.

12. Wen HY, Brogi E. Lobular Carcinoma In Situ. Surgical Pathology Clinics. 2018;11(1):123-45. doi: 10.1016/j.path.2017.09.009.

13. Srivastava S. Breast noninvasive lobular neoplasia: LCIS classic, 2021 [Access date: November 11th 2021. Available from:

ttps://www.pathologyoutlines.com/topic/breastmalign antlcis.html.

14. Savage JL, Jeffries DO, Noroozian M, Sabel MS, Jorns JM, Helvie MA. Pleomorphic Lobular Carcinoma In Situ: Imaging Features, Upgrade Rate, and Clinical Outcomes. AJR Am J Roentgenol. 2018;211(2):462-7. doi: 10.2214/AJR.17.19088.

\section{How to Cite This Article}

Pang FT, Kornecki A, Lynn K, Lee SY. A Rare Case of Lobular Carcinoma in Situ Within a Fibroadenoma. Arch Breast Cancer. 2022; 9(1):119-122.

Available from: https://www.archbreastcancer.com/index.php/abc/article/view/432 